\title{
Introduction of the MyoSureLITE in an established outpatient hysteroscopy clinic
}

Hannah G GORDON ${ }^{1,2}$ (Obstetrics and Gynaecology Resident)

Samantha MOONEY² (Endosurgery Fellow; samantha.mooney39@gmail.com)

Emma READMAN ${ }^{1,2}$ (Endosurgery Consultant; ereadman@melbpc.org.au)

${ }^{1}$ University of Melbourne, Department of Obstetrics and Gynaecology, Parkville, Australia.

${ }^{2}$ Mercy Hospital for Women, 163 Studley Road, Heidelberg, Australia.

\section{Corresponding author:}

Dr Hannah G Gordon

ORCID 0000-0001-7510-0071

Postal address: Department of Obstetrics and Gynaecology,

Faculty of Medicine, Dentistry and Health Sciences,

University of Melbourne, Building 181

Grattan St, Melbourne 3010

Email: hannah-gordon@outlook.com

\section{Conflicts of interest:}

ER has previously done unpaid consulting for Hologic. Neither HGG nor SM have conflicts of interest to declare.

This is the author manuscript accepted for publication and has undergone full peer review but has not been through the copyediting, typesetting, pagination and proofreading process, which may lead to differences between this version and the Version of Record. Please cite this article as doi: $10.1111 / \mathrm{AJ} 0.13207$

This article is protected by copyright. All rights reserved 


\section{Unblinded sentences:}

\section{Materials and Methods}

"The outpatient hysteroscopy (OPH) clinic at the Mercy Hospital for Women was established in 1993, with a prospective database started in 2003."

\section{Discussion}

"Ma et al found that between 2003 and 2014, the prevalence of endometrial polyps in women presenting to the Mercy Hospital OPH clinic was $14.1 \%$."

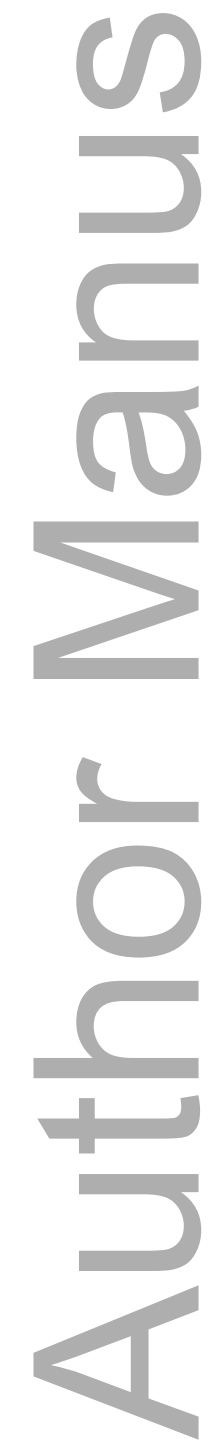


DR. HANNAH G GORDON (Orcid ID : 0000-0001-7510-0071)

DR. SAMANTHA SCOTT MOONEY (Orcid ID : 0000-0001-5742-9148)

Article type : Original Manuscript

\section{口 \\ Introduction of the MyoSureLITE in an established outpatient hysteroscopy clinic}

Running title: MyoSureLITE in an established OPH clinic

Keywords: hysteroscopy; outpatient clinics; polyps; abnormal uterine bleeding; uterine neoplasms

Manuscript word count: 2504 words

Abstract word count: 240 words

Figure count: 1

Table count: 3

Abstract

Background: Endometrial polyps are a common cause of abnormal uterine bleeding. The MyoSureLITE intrauterine morcellation device is effective at resecting endometrial polyps, however its use in the outpatient setting requires appraisal.

Aim: To assess the feasibility, utility, acceptability and costs associated with introduction of the MyoSureLITE into an established outpatient hysteroscopy (OPH) clinic. 
Material and Methods: A prospective clinical database from a tertiary Melbourne hospital was analysed from 1 July 2015 to 30 June 2018. Three 12-month time periods were compared; pre-introduction and trial phase, early use, and established use of the MyoSureLITE. Wait times, patient acceptability, second OPH bookings and procedure costs were measured.

Results: 871 women underwent OPH during the study period, with 238(27.3\%) women presenting with endometrial polyp(s). At each timepoint, $78.5 \%, 25 \%$ and $6.3 \%$ of women required re-booking for a subsequent hysteroscopy for pathology otherwise suitable for MyoSureLITE resection. Introduction of the MyoSureLITE avoided a subsequent procedure for 4, 60 and 69 women respectively for each year of use, with potentially reduced treatment costs for the institution.

Median wait-time for definitive treatment of intrauterine pathology was 56[24-84] days at timeperiod 1 , decreasing to $0[0-0]$ days during time-period 3 , $(\mathrm{p}<0.001) .87 .6 \%$ would undergo $\mathrm{OPH}$ again.

Conclusions: Routine use of the MyoSureLITE is effective, feasible, and acceptable to women. Provision of this device in outpatient service allows a 'see and treat' model, saving theatre time and treatment costs, facilitating a more direct throughput from presentation to treatment.

\section{Introduction}

Endometrial polyps are a common cause of abnormal uterine bleeding in pre- and postmenopausal women. ${ }^{1}$ The rate of spontaneous regression of endometrial polyps is low, ${ }^{2}$ and while the levonorgestrel intrauterine device might have an adjunct role in treating polyps among women with heavy menstrual bleeding, ${ }^{3}$ because of the associated relationship between endometrial polyps and endometrial cancer, ${ }^{4}$ current guidelines recommend complete resection of the polyp with an endometrial biopsy of the surrounding area. ${ }^{5}$

The MyoSureLITE® (Hologic, Marlboro MA) is an intrauterine morcellation device used to resect endometrial polyps in an inpatient or outpatient setting. Rubino et al have demonstrated that endometrial polyp and/or fibroid resection using the MyoSure confers a significant improvement in quality of life for up to 12 months among women experiencing abnormal uterine bleeding due to these pathologies. ${ }^{6}$ Intrauterine morcellation is also associated with a 
lower recurrence of endometrial polyps compared with initial resection via traditional operative polypectomy. ${ }^{7}$

The outpatient clinic has been well established as a safe, cost effective and acceptable mode for hysteroscopy, with high rates of patient satisfaction, and faster recovery times when compared with inpatient hysteroscopy, avoiding the need for general anaesthetic. ${ }^{8-10}$ But, in Australia, larger studies evaluating the effectiveness of the MyoSure have only been performed in an inpatient setting due to lack of appropriate government reimbursement for outpatient treatment despite the potential cost-benefit of the procedure. ${ }^{11}$

Specific to the outpatient setting, previous research has suggested that the MyoSure is efficient, safe and acceptable to use for resection of endometrial polyps, but this research is limited by small sample sizes and a cross-sectional approach. ${ }^{9,12}$ This paper, by comparison, aims to assess the utility, acceptability and cost benefit of the MyoSureLITE across the first three years of its introduction into an established outpatient hysteroscopy clinic.

\section{Materials and Methods}

The outpatient hysteroscopy (OPH) clinic at the Mercy Hospital for Women (Melbourne, Australia) was established in 1993, with a prospective database started in 2003. Indications for referral to the outpatient hysteroscopy clinic include abnormal uterine bleeding, assessment of intrauterine abnormalities demonstrated on imaging, or insertion or removal of an intra-uterine device. Patients considered for OPH must be medically capable of tolerating a vasovagal response and accordingly, relative contraindications include age $>65$ years, weight $>120 \mathrm{~kg}$ and significant cardiac comorbidities. Moreover, women who are expected to tolerate the procedure poorly, such as those who report an inability to tolerate a vaginal examination, or women who are unwilling to undergo the procedure while awake are booked for an inpatient procedure under general anaesthetic.

Prior to the introduction of the MyoSure, all women with suspected endometrial pathology on ultrasound would require initial OPH to confirm the presence of a polyp, with subsequent booking as an inpatient for resection. The MyoSureLITE was first available for use in the clinic in May 2016. 
A subsection of the database was analysed from 1 July 2015 to 30 June 2018, considering only women presenting to the clinic with endometrial polyps. Each entry includes information about patient age, menopausal status, previous vaginal delivery (yes/no), ultrasound findings and the reason for presenting to the OPH clinic. Verbal consent was obtained from all participants for inclusion in the database. This paper is consistent with a quality assurance audit, with no additional risk to patients. In line with National Health and Medical Research Council guidelines, ${ }^{13}$ and consistent with similar published studies using the same database, ${ }^{10}$ formal institutional ethics approval was not required.

Three 12-month time periods were compared; pre-introduction and trial phase of the MyoSureLITE (July 2015-June 2016), early use of the MyoSureLITE (July 2016-June 2017) and established use (June 2017-July 2018). Endometrial polyp resection using the MyoSureLITE was performed by all levels of clinicians from registrar through to consultant.

\section{MyoSureLITE Technique}

Upon admission, women are offered diclofenac $50 \mathrm{mg}$ unless contraindicated, in which case paracetamol $1 \mathrm{~g}$ is used. A 3mm Olympus® (Olympus Australia, Melbourne, Victoria, Australia) rigid hysteroscope with a $4 \mathrm{~mm}$ sheath is passed through the vagina and cervical canal into the uterine cavity using saline as a distension medium. If an endometrial polyp is noted, a bi-valved speculum is used to visualise the cervix, and $6-10 \mathrm{~mL}$ of $2 \%$ lignocaine with adrenaline is administered as a paracervical block. The cervix is dilated to Hagar 6. The MyoSure Hysteroscope (outer diameter $6 \mathrm{~mm}$ ) provides the operating channel for the MyoSureLITE device, as well as continuous fluid inflow/outflow. The polyp is resected under vision and the tissue is sent for histology.

Utility

Utility was determined by: (1) The median number of days between OPH clinic appointment (at which the presence of an endometrial polyp was confirmed) and definitive treatment.

(2) Whether or not second hysteroscopic procedures were required. This included repeat OPH clinic appointments due to MyoSure device unavailability, and inpatient hysteroscopic procedures requiring general anaesthetic.

Acceptability 
Acceptability was assessed using a Visual Analogue Score (VAS) for pain experienced during the procedure, from 0 (no pain) to 10 (significant pain). Women were also asked whether they would repeat the procedure in the future, if necessary (Yes/No). Any complications encountered during the procedure were documented.

\section{Cost impacts}

Costs of outpatient versus inpatient hysteroscopy were estimated after discussion with the hospital finance team. Costs were estimated by summating and comparing the costs associated with (1) outpatient and (2) inpatient hysteroscopic polyp resection. These costs included personnel, technology, pharmacy, pathology, and loss of income for the patient. Loss of income for the patient was estimated by using women's average weekly earnings as calculated by the Australian Bureau of Statistics. ${ }^{14}$

\section{Statistical analysis}

Data was collected using an Access (Microsoft 2018) database platform. Statistical analysis was performed using both Excel (Microsoft 2018) and STATA Data Analysis and Statistical Software v14 (StataCorp, College Station, TX, USA). Results are expressed as a mean (SD), median $[\mathrm{IQR}]$ or percentage of a total. A one-way ANOVA was performed to determine whether there was a significant difference between mean patient age across the three time periods. A Fisher's exact test was used to determine whether there was a significant difference in menopausal status and vaginal parity across the three time periods, and a Chisquare test to determine whether there was a significant difference in reason for presentation to the clinic across time periods. A Wilcoxon Rank Sum Test was performed to determine whether the difference in waiting times between time period one and time period three was significant. P-values $<0.05$ were considered statistically significant.

\section{Results}

During the study period, 871 women underwent outpatient hysteroscopy, and 238 (27.3\%) of these women were identified as having endometrial polyps. Of women with endometrial polyps, the mean [SD] age was 48.6 [9.6], 48.8 [9.1] and 49.1 [10.1] years at each time-period respectively (Table 1). The majority of women presenting to the clinic were pre- or perimenopausal, and had not had a previous vaginal delivery. Patient age, menopausal status, vaginal parity, and reason for presenting to the $\mathrm{OPH}$ clinic did not change significantly across the time period. 


\section{Utility}

In the first year of the introduction of the MyoSureLITE, 94.2\% ( $n=65)$ of women presenting with endometrial polyps required re-booking for definitive management as an inpatient $(n=57)$ or an outpatient $(n=8)$ (Table 2). Comparatively, in time-period two, 32.9\% ( $n=28)$ of women required rebooking. Nine of these patients required rebooking for repeat $\mathrm{OPH}$ appointments due to MyoSureLITE unavailability at the initial consult, and seven of these women were subsequently successfully managed with the MyoSureLITE (Figure 1).

During time-period three, $19.0 \%(\mathrm{n}=16)$ of women required rebooking, with only one of these 16 suitable for a repeat outpatient procedure. The other women required inpatient procedures due to other pathologies, such as concurrent fibroids not suitable for MyoSureLITE resection $(n=5)$, pain secondary to cervical stenosis $(n=9)$ and request for alternative treatment $(n=1)$.

Widespread availability of the MyoSureLITE allowed for a 'see and treat' approach, with resection of endometrial polyps at initial OPH appointment, therefore avoiding a subsequent procedure for 4,59 and 68 women respectively for each year of use. Among women presenting with endometrial polyps, complete resection was achieved in $98.5 \%$ of cases (129 out of 131 cases). Both cases needing re-resection were initially performed by consultants.

Median [IQR] wait time from the first OPH clinic to definitive treatment of endometrial polyp decreased from 56 [24-84] days at time-period 1 to 0 [0-0] days at time-period 3 ( $\mathrm{p}<0.001)$

(Table 3). In all three groups there were significant outliers in the $4^{\text {th }}$ quartile, with the maximum waiting times of 332, 402, and 203 days in time-periods 1 to 3 respectively.

Acceptability

Among women with endometrial polyps where the MyoSureLITE was used for resection, mean [SD] pain scores out of ten were 7.3 [0.5] during time-period 1, 4.5 [2.8] during time-period 2 and 5.0 [2.9] during time-period 3. Once the Myosure was well-established in the clinic (timeperiods 2 and 3), $87.6 \%$ of patients reported that they would undergo the outpatient MyoSure procedure again. Median $[\mathrm{IQR}]$ pain scores among patients who would repeat the procedure were $5[3,6]$, compared with those who would not repeat the procedure, $8.5[6,10]$. 
No serious complications were encountered by any patients across the study period. Eight women $(3.4 \%)$ were recorded as experiencing a pre-syncopal or vasovagal response during the procedure.

Cost impacts

Despite the MyoSureLITE device costing approximately double that of the bipolar electrode (used for hysteroscopic resection of polyps in theatre), it was calculated that the additional cost of theatre time, nursing staff, anaesthetic team, pharmacy, and other consumables amounted to more than $\$ 2000$ more per case performed in the operating theatre under general anaesthetic. Cost estimates from 2019 local hospital finance data indicate that inpatient hysteroscopic resection of polyps cost approximately AUD\$2945.00 per case, compared with OPH with MyoSureLite which costs approximately AUD\$880.00. At present, there is no difference in the revenue received by public institutions regardless of whether the woman undergoes an inpatient- or outpatient procedure.

In the Australian public health system, the costs of both inpatient and outpatient hysteroscopic procedures are covered by Medicare, and thus the patient incurs no out-of-pocket costs for these procedures. However, for the patient, an inpatient hysteroscopy represents the loss of two days ( AUD\$600) income, or two days paid leave for the employer. ${ }^{14}$ For the procedure under a general anaesthetic, women are required to take leave from employment on both the day of surgery, as well as for 24 hours after. Furthermore, a carer is required to assist the woman home post-operatively, likely contributing to additional leave from employment. Conversely, in the outpatient setting, women undergoing an outpatient hysteroscopy usually attend work on the morning of their procedure, and can return to work the morning following their procedure. This represents a $\sim$ AUD $\$ 150$ loss of income for the patient, a quarter of that lost with the inpatient general anaesthetic option. ${ }^{14}$ These women do not need an escort to or home from the procedure.

In addition to this monetary cost savings, the opportunity cost cannot be understated. Managing these women as outpatients frees up theatre time for major gynaecological cases, allowing timely care for women who require inpatient care.

\section{Discussion}


Ma et al found that between 2003 and 2014, the prevalence of endometrial polyps in women presenting to the Mercy Hospital OPH clinic was $14.1 \% .{ }^{8}$ Using the same database, this study has observed a two-fold increase in the prevalence of women presenting with endometrial polyps to the outpatient clinic in recent years. Detection of endometrial polyps on ultrasound may have previously indicated referral for inpatient hysteroscopy, resulting in a large number of women bypassing the OPH clinic.

Women were generally accepting of the procedure, outpatient endometrial polyp resection with MyoSure with $87.6 \%$ reporting they would undergo the procedure again if necessary during time-periods 2 and 3. Satisfaction rates were however not as high as acceptability of OPH by Georgiou et al (95.2\%) and McIlwaine et al (99\%). ${ }^{12,15}$ Median pain scores were also higher among patients who reported they would not repeat the procedure in future, suggesting that pain is likely to be a factor contributing to a patient's perception of the OPH experience.

The implementation of the MyoSure resulted in a significant reduction in median waiting times between initial presentation and definitive management.

The cost impacts are persuasive and supported by further recent data in another Australian setting. ${ }^{16}$ Our study demonstrates that see-and-treat management of endometrial polyps with the MyoSureLITE device in the outpatient is less costly compared to the equivalent inpatient procedure.

This study had a number of strengths. Whilst similar previous studies have had one or two surgeons performing cases, this study included cases performed by clinicians of all levels of expertise with outcomes measured longitudinally rather than cross-sectionally. ${ }^{12}$ While Arnold et al noted that in the inpatient setting, junior doctors were less competent at completely resecting endometrial polyps, this study found that there was no apparent difference in resection rates between registrars, fellows and consultants. ${ }^{10}$

\section{Limitations}

Inherent to database research design, there is the possibility for error during data entry, or for incomplete data entry. There was inconsistency in recording the time it took for the MyoSureLITE to be used to resect polyps such that this variable has been excluded from results. Further, there was limited past medical history documented for each patient, and 
accordingly it is unclear whether there was any significant change in comorbidities across the three groups.

Capital costs were not included in the cost calculations for this analysis, other than the use of the disposable MyoSureLITE device itself. The unit's MyoSure-compatible hysteroscopes were loaned from the manufacturer, as was the electrical unit for the Myosure. However, should a unit need to purchase these scopes, they are of similar costing to the operative hysteroscopes used in the operating theatre, and thus should not alter our cost comparisons between the two techniques. The hysteroscopic stack was previously owned by the hospital, and would be of equivalent or cheaper value compared to the stacks in the operating theatre and thus not helpful in the comparison costs. A procedure chair is required for outpatient hysteroscopy procedures. Our institution was already in possession of this chair, which was also used for outpatient cystoscopic procedures and anal manometry. Again, the cost of this chair was not included in the analysis as it was already owned by the hospital, and is less costly than a theatre operating table. However, for units planning to establish a new outpatient hysteroscopy service equipped with MyoSure capabilities, these capital costs need to be evaluated. A formal cost-benefit analysis is planned to provide additional support for the cost-effectiveness of outpatient polypectomy with MyoSureLITE. A formal cost-benefit analysis is planned to provide additional support for the cost-effectiveness of outpatient polypectomy with MyoSure LITE.

It would be interesting to note whether size of the polyp impacted on the likelihood of resection, however, in the majority of cases, polyps were received by the histopathology department in fragments, making it difficult to determine this. This could be explored in future research.

\section{Conclusion}

Routine use of the MyoSureLITE is effective, feasible, safe, and acceptable to women. Provision of this device in outpatient service saves theatre time and treatment costs and allows a more direct throughput from presentation to treatment.

\section{References}

1. Dreisler E, Stampe Sorensen S, Ibsen PH, Lose G. Prevalence of endometrial polyps and abnormal uterine bleeding in a Danish population aged 20-74 years. Ultrasound Obstet Gynecol. 2009 Jan 1;33(1):102-8. 
2. Lieng M, Istre O, Sandvik L, Qvigstad E. Prevalence, 1-Year Regression Rate, and Clinical Significance of Asymptomatic Endometrial Polyps: Cross-sectional Study. J Minim Invasive Gynecol. 2009 Jul 1;16(4):465-71.

3. Chowdary P, Maher P, Ma T, Newman M, Ellett L, Readman E. The Role of the Mirena Intrauterine Device in the Management of Endometrial Polyps: A Pilot Study. J Minim Invasive Gynecol. 2019;26(7):1297-302.

4. Savelli L, De Iaco P, Santini D, Rosati F, Ghi T, Pignotti E, et al. Histopathologic features and risk factors for benignity, hyperplasia, and cancer in endometrial polyps. Am J Obstet Gynecol. 2003;188(4):927-31.

5. Gallos I, Alazzam M, Clark T, Faraj R, Rosenthal A, Smith P, et al. Management of Endometrial Hyperplasia. Green-top Guideline No. 67. 2016;

6. Rubino RJ, Lukes AS. Twelve-month outcomes for patients undergoing hysteroscopic morcellation of uterine polyps and myomas in an office or ambulatory surgical center. $\mathrm{J}$ Minim Invasive Gynecol. 2015;22(2):285-90.

7. AlHilli MM, Nixon KE, Hopkins MR, Weaver AL, Laughlin-Tommaso SK, Famuyide AO. Long-term outcomes after intrauterine morcellation vs hysteroscopic resection of endometrial polyps. J Minim Invasive Gynecol. 2013;20(2):215-21.

8. Kremer C, Duffy S, Moroney M. Patient satisfaction with outpatient hysteroscopy versus day case hysteroscopy: randomised controlled trial. Bmj. 2000;320(7230):279-82.

9. McIlwaine P, McElhinney B, Karthigasu KA, Hart R. A prospective study of the use of the Myosure resectoscope to manage endometrial polyps in an outpatient setting. Aust $\mathrm{N}$ Z J Obstet Gynaecol. 2015 Oct 1;55(5):482-6.

10. Ma T, Readman E, Hicks L, Porter J, Cameron M, Ellett L, et al. Is outpatient hysteroscopy the new gold standard? Results from an 11 year prospective observational study. Aust N Z J Obstet Gynaecol. 2017;57(1):74-80.

11. Arnold A, Ketheeswaran A, Bhatti M, Nesbitt-Hawes E, Abbott J. A prospective analysis of hysteroscopic morcellation in the management of intrauterine pathologies. J Minim Invasive Gynecol. 2016;23(3):435-41. 
12. Georgiou D, Tranoulis A, Jackson TL. Hysteroscopic tissue removal system (MyoSure) for the resection of polyps, sub-mucosal leiomyomas and retained products of conception in an out-patient setting: A single UK institution experience. Eur J Obstet Gynecol Reprod Biol. 2018;231:147-51.

13. NHMRC. National Health and Medical Research Council. Canberra; 2007.

14. Australian Bureau of Statistics. Average Weekly Earnings, Australia, cat. no. 6302.0 [Internet]. 2019. Available from:

https://www.abs.gov.au/ausstats/abs@.nsf/mf/6302.0?opendocument\&ref=HPKI

15. McIlwaine K, Readman E, Cameron M, Maher P. Outpatient hysteroscopy: Factors influencing post-procedure acceptability in patients attending a tertiary referral centre. Aust N Z J Obstet Gynaecol. 2009;49(6):650-2.

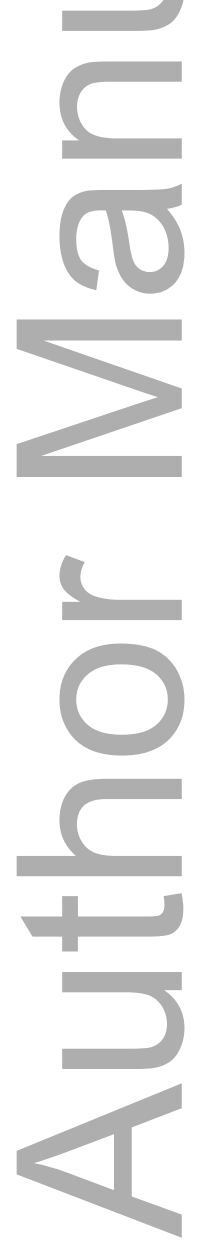


Table 1.

\begin{tabular}{|c|c|c|c|c|c|}
\hline \multicolumn{6}{|c|}{ Demographic characteristics } \\
\hline & & $\begin{array}{l}\text { Time period } \\
\text { 1: } 2015-2016\end{array}$ & $\begin{array}{l}\text { Time period } \\
\text { 2: 2016-2017 }\end{array}$ & $\begin{array}{l}\text { Time period } \\
\text { 3: 2017-2018 }\end{array}$ & $\mathbf{p}$ \\
\hline \multicolumn{2}{|c|}{ Age in years, mean [SD] } & $48.6[9.6]$ & $48.8[9.1]$ & $49.1[10.1]$ & $0.950^{*}$ \\
\hline \multirow{2}{*}{$\begin{array}{l}\text { Menopausal status, } \mathrm{n} \\
(\%)\end{array}$} & Pre- or perimenopausal & $44(63.8 \%)$ & $52(61.2 \%)$ & $51(60.7 \%)$ & \multirow[t]{2}{*}{$0.943^{* *}$} \\
\hline & Postmenopausal & $25(36.2 \%)$ & $33(38.8 \%)$ & $33(39.3 \%)$ & \\
\hline \multirow[t]{2}{*}{ Vaginal parity, n (\%) } & 0 & $48(69.6 \%)$ & $56(65.9 \%)^{\dagger}$ & $59(70.2 \%)$ & \multirow[t]{2}{*}{$0.937^{* *}$} \\
\hline & $\geq 1$ & $21(30.4 \%)$ & $27(31.8 \%)^{\dagger}$ & $25(29.8 \%)$ & \\
\hline \multirow{4}{*}{$\begin{array}{l}\text { Clinical presentation, } \\
\mathrm{n}(\%)\end{array}$} & Postmenopausal bleeding & $14(20.3 \%)$ & $21(24.7 \%)$ & $20(23.8 \%)$ & \multirow[t]{4}{*}{$0.219^{* * * *}$} \\
\hline & Heavy menstrual bleeding & $34(49.3 \%)$ & $25(29.4 \%)$ & $25(29.8 \%)$ & \\
\hline & Intermenstrual bleeding & $6(8.7 \%)$ & $13(15.3 \%)$ & $13(15.5 \%)$ & \\
\hline & Other & $15(21.7 \%)$ & $26(30.6 \%)$ & $26(30.9 \%)$ & \\
\hline
\end{tabular}

Table 2.

\begin{tabular}{|l|c|c|c|}
\hline \multicolumn{1}{|l|}{ Introduction of the MyoSureLITE } & $\begin{array}{c}\text { Time-period 1: } \\
\mathbf{2 0 1 5 - 2 0 1 6}\end{array}$ & $\begin{array}{c}\text { Time-period 2: } \\
\mathbf{2 0 1 6 - 2 0 1 7}\end{array}$ & $\begin{array}{c}\text { Time-period 3: } \\
\mathbf{2 0 1 7 - 2 0 1 8}\end{array}$ \\
\hline & 69 & 85 & 84 \\
\hline Endometrial polyps diagnosed at OPH & 4 & 59 & 68 \\
\hline $\begin{array}{l}\text { Attempted OPH-resection using } \\
\text { MyoSureLITE, } n\end{array}$ & $4(100 \%)$ & $57(96.6 \%)$ & $68(100 \%)$ \\
\hline $\begin{array}{l}\text { Successful OPH-resections using } \\
\text { MyoSureLITE, } n \text { (\%) }\end{array}$ & 65 & $288^{*}$ & 16 \\
\hline $\begin{array}{l}\text { Total number of patients needing re- } \\
\text { booking (any reason), } n\end{array}$ & $51(78.5 \%)$ & $7(25 \%)$ & $1(6.3 \%)$ \\
\hline $\begin{array}{l}\text { Second procedures that could have been } \\
\text { avoided with routine availability of the } \\
\text { MyoSureLITE, } n \text { (\% of rebooked) }\end{array}$ & & & \\
\hline
\end{tabular}

Table 3.

\begin{tabular}{|l|c|c|c|c|}
\hline \multicolumn{6}{|c|}{ Acceptability of the MyoSureLITE among patients with endometrial polyps } \\
\hline & Time-period 1: & Time-period 2: & Time-period 3: & P value \\
& $2015-2016$ & $2016-2017$ & $2017-2018$ & \\
\hline
\end{tabular}

This article is protected by copyright. All rights reserved 


\begin{tabular}{|l|c|c|c|c|}
\hline $\begin{array}{l}\text { Days to definitive polyp } \\
\text { treatment, median [IQR] }\end{array}$ & $56[24-84]$ & $0[0-6]$ & $0[0-0]$ & $<0.001^{*}$ \\
\hline Procedure pain score, mean [SD] & $7.3[0.5]$ & $4.5[2.8]$ & $5.0[2.9]$ & N/A $^{* *}$ \\
\hline
\end{tabular}

${ }^{*}$ Wilcoxon Rank-Sum Test

${ }^{* *}$ Not performed due to small sample size $(\mathrm{N}=4)$ in Time-period 1

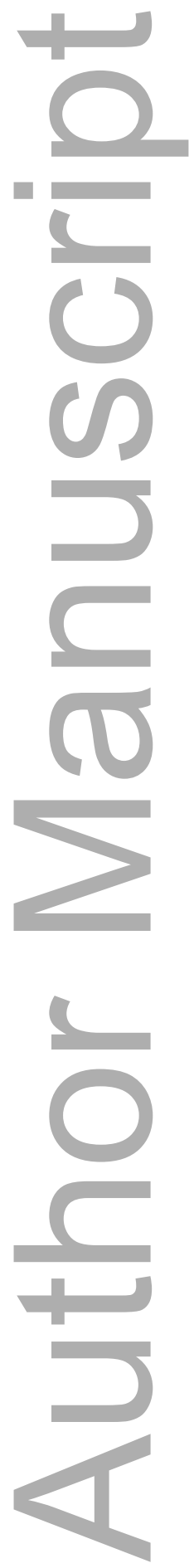




\section{All OPH attendances 1 July 2015}

30 June 2018, n=871

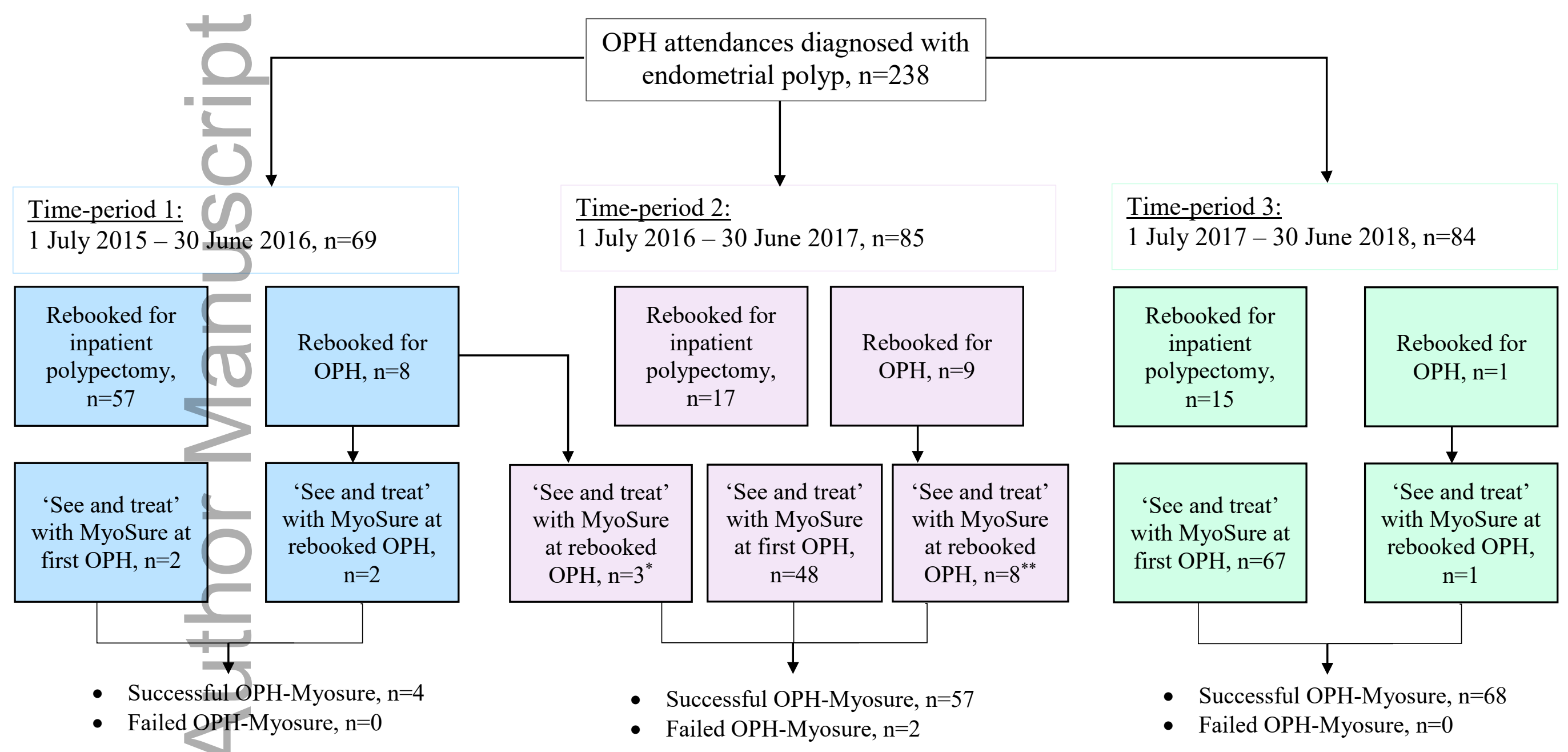

*Three patients did not attend repeat OPH appointment

${ }^{* *}$ One patient did not attend repeat OPH appointment

Figure 1: Participant flow chart

This article is protected by copyright. All rights reserved 


\section{University Library}

\section{- M M I N E R VA A gateway to Melbourne's research publications}

Minerva Access is the Institutional Repository of The University of Melbourne

Author/s:

Gordon, HG;Mooney, S;Readman, E

Title:

Introduction of the MyoSureLITE in an established outpatient hysteroscopy clinic

Date:

2020-07-22

Citation:

Gordon, H. G., Mooney, S. \& Readman, E. (2020). Introduction of the MyoSureLITE in an established outpatient hysteroscopy clinic. AUSTRALIAN \& NEW ZEALAND JOURNAL OF OBSTETRICS \& GYNAECOLOGY, 60 (5), pp.784-789. https://doi.org/10.1111/ajo. 13207.

Persistent Link:

http://hdl.handle.net/11343/276039 